\section{Biopsia por punción y prostatectomía radical}

\section{Trans rectal biopsies and radical prostatectomy}

\section{Sr Editor:}

Con mucho interés hemos leído el artículo de Tapia y cols titulado "Puntaje de Gleason en cáncer de próstata: Correlación de la biopsia por punción y prostatectomía radical"'. En este estudio retrospectivo de 168 pacientes los autores describen $68 \%$ de correlación entre la biopsia transrectal y la pieza quirúrgica post prostatectomía radical. Asimismo, los autores encontraron que en $28 \%$ de los casos la biopsia de la pieza quirúrgica demostró un adenocarcimona más agresivo que aquel originalmente reportado en la biopsia transrectal. Es sabido que el cáncer de próstata puede tener un curso agresivo así como también uno indolente, el que rara vez comprometerá la vida del enfermo. Estudios internacionales han demostrado que en alrededor de 30\% de los pacientes en los que la biopsia transrectal sugería un tumor indolente, finalmente se confirmó un tumor agresivo al momento de estudiar la pieza operatoria post prostatectomía radical ${ }^{2}$. Esta incapacidad de la biopsia transrectal de determinar con certeza la agresividad de un cáncer prostático es un gran problema para los urólogos al momento de aconsejar a un paciente entre el tratamiento curativo y la observación activa de su enfermedad. En los últimos años se han desarrollado métodos de diagnóstico que podrían ayudar a diferenciar entre un cáncer agresivo o uno indolente. La resonancia nuclear magnética multiparamétrica combina las características fisico-químicas del tejido, como la difusión y espectrometría, y se ha mostrado promisoria en determinar la agresividad de un tumor ${ }^{3}$. La medición de la proteína PCA3 (prostate cancer gene 3) permite diferenciar entre tejido canceroso y glándulas benignas, y el nivel detectado en orina se ha asociado directamente con la agresividad del tumor ${ }^{4}$. Por último, una mayor cantidad de receptores de andrógenos en un cilindro de biopsia transrectal (Aureon test) se ha asociado con el riesgo de poseer un tumor de mayor agresividad 5 .

Desgraciadamente, aún no es posible diferenciar con exactitud qué paciente con cáncer prostá- tico porta un tumor agresivo o uno indolente. Sin duda que esa incertidumbre contribuye a que en ciertas ocasiones se sobre-indique una intervención quirúrgica. Afortunadamente, actualmente existen técnicas quirúrgicas menos invasivas, como la prostatectomía robótica, la que en manos de un cirujano con experiencia ofrece excelentes resultados oncológicos y funcionales ${ }^{6}$.

\section{Drs. Oscar Schatloff y Vipul Patel Global Robotics Institute, USA.}

\section{Referencias}

1. Tapia OE, Bellolio EJ, Roa JC, Guzmán PG, Villaseca $\mathrm{MH}$, Araya JO. Puntaje de Gleason en cáncer de próstata: Correlación de la biopsia por punción y prostatectomía radical. Rev Med Chile 2011; 139: 171-6.

2. Iremashvili V, Manoharan M, Pelaez L, Rosenberg DL, Soloway MS. Clinically significant Gleason sum upgrade: External validation and head-to-head comparison of the existing nomograms. Cancer. 2011 Jul 5. doi: 10.1002/ cncr.26306. [Epub ahead of print].

3. Rouse P, Shaw G, Ahmed HU, Freeman A, Allen C, Emberton M. Multi-Parametric Magnetic Resonance Imaging to Rule-In and Rule-Out Clinically Important Prostate Cancer in Men at Risk: A Cohort Study. Urol Int. 2011 Jun 22. [Epub ahead of print].

4. Rubio-Briones J, Fernández-Serra A, Ramírez M, Rubio L, Collado A, Casanova J, et al. Outcomes of Expanded Use of PCA3 Testing in a Spanish Population with Clinical Suspicion of Prostate Cancer. Actas Urol Esp. 2011 Jun 21. [Epub ahead of print].

5. Donovan MJ, Khan FM, Fernández G, Mesa-Tejada R, Sapir M, Zubek VB, et al. Personalized prediction of tumor response and cancer progression on prostate needle biopsy. J Urol 2009; 182: 125-32.

6. Patel VR, Coelho RF, Chauhan S, Orvieto MA, Palmer $\mathrm{KJ}$, Rocco B, et al. Continence, potency and oncological outcomes after robotic-assisted radical prostatectomy: early trifecta results of a high-volume surgeon. BJU Int. 2010; 106: 696-702. orrespondencia a:

Dr. Oscar Schatloff.

E-mail oscar.schatloff@gmail.com

Los autores declararon no tener conflictos de intereses en relación al tema de su Carta al Editor. 